\title{
PARSIMONY IN NON-ZERO SECONDARY LOADINGS IN CONFIRMATORY FACTOR ANALYSIS
}

\author{
ANDRÉ BEAUDUCEL \\ Institute of Psychology \\ University of Bonn \\ Kaiser-Karl-Ring 9 \\ 53111 Bonn \\ Germany \\ e-mail: beauducel@uni-bonn.de
}

\begin{abstract}
In the context of confirmatory factor analysis, the independent clusters model (ICM), a model with non-salient loadings that are fixed to zero, is often tested. However, the ICM has been found to be overly restrictive in several research contexts. The aim of the present study was therefore to propose a less restrictive criterion for parsimony of non-salient loadings. A promising criterion for this purpose is based on 'buffered scales', which have been proposed by Cattell and Tsujioka [1] as optimal indicators of corresponding factors. Variables with positive and negative loadings on an unwanted factor are balanced out in a buffered scale, so that the variance of the unwanted factor is at minimum. Model constraints are proposed in order to specify the balance of positive and negative loadings on unwanted factors as a basis for loading parsimony.
\end{abstract}

2010 Mathematics Subject Classification: 62H25.

Keywords and phrases: confirmatory factor analysis, independent clusters model, model constraints.

Received May 10, 2017

(C) 2017 Scientific Advances Publishers 


\section{Introduction}

In confirmatory factor analysis (CFA), the salient factor loadings are typically freely estimated, whereas the loadings of the variables that are not expected to load on the respective factors are often fixed to zero. In the following, the largest absolute loadings that are used for the interpretation of the factors are termed 'salient' loadings, whereas those absolute loadings that are considerably smaller than the salient loadings and that are usually not used for the interpretation of the factors are termed 'non-salient' or 'secondary' loadings. In the following, a model that is based on variables with only one salient loading and with all nonsalient loadings being fixed to zero will be termed independent clusters model (ICM). The fixation of non-salient loadings to be zero in the ICM has been criticized because the specification of zero-loadings is often more strict than should be expected from theory (Hofstee et al. [2]; Vassend \& Skrondal [3]).

In the ICM one would expect that the absolute loadings of items on a factor are either very high or zero. In contrast, Cattell and Tsujioka [1] introduced the idea of 'buffered scales' based on items with positive loadings on an intended factor and a balanced set of positive and negative loadings on an unwanted factor. An example of two items with positive loadings on the wanted factor and with a balanced set of positive and negative loadings on the unwanted factor is given in Figure 1(A). In contrast to the ICM, the absolute size of the loadings on the unwanted factor is large whereas the sign of the loadings on the unwanted factor is reversed. One might consider

$$
\min _{l_{11}, \ldots, l_{k 1}}\left(\sum_{i=1}^{k} l_{i 1}\right)^{2},
$$

where the subscript " 1 " indicates the first column of the factor loading matrix and $k$ is the number of non-salient loadings in the first column of the loading matrix, and the subscript " $i$ " indicates the respective number of the non-salient loading. Moreover, finding the balance of positive and negative non-salient loadings on the wanted factor should take the loading size on the unwanted factor into account (see Figure 1(B)). 
(A)

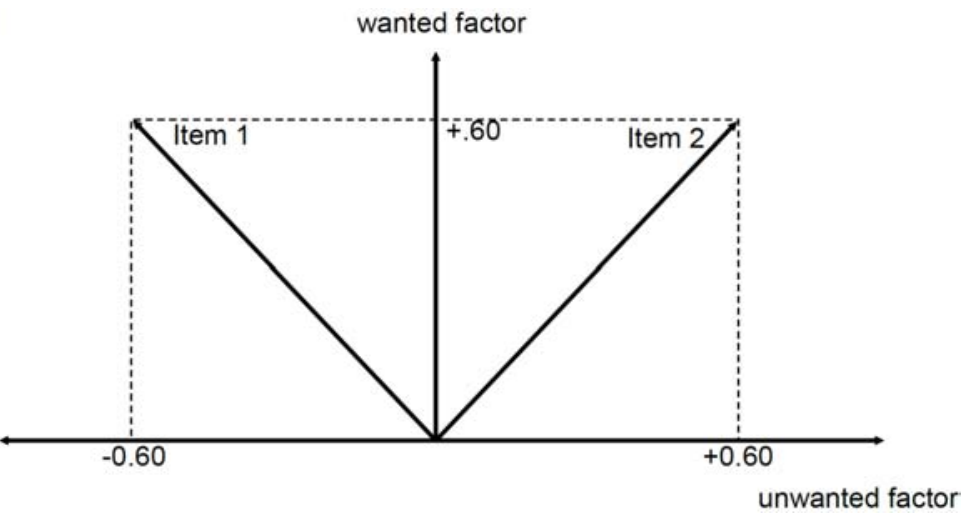

(B)

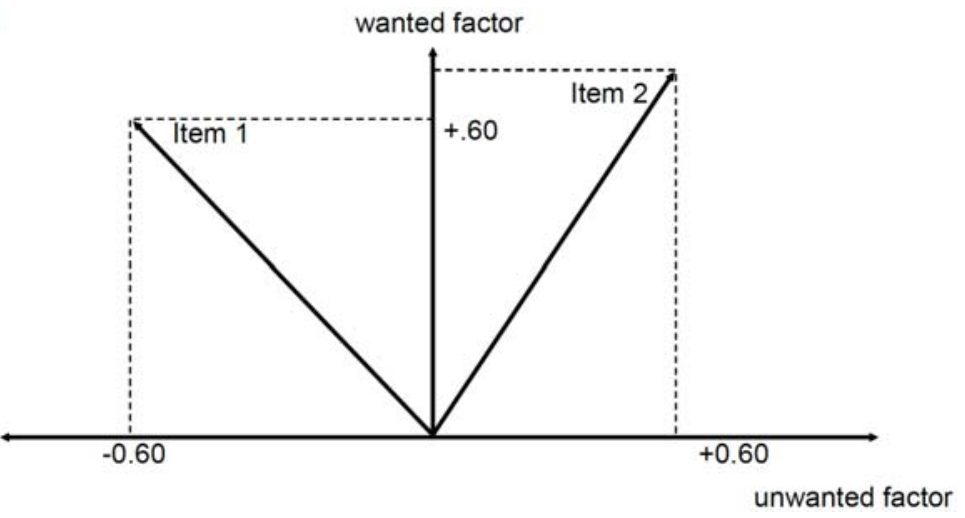

(C)

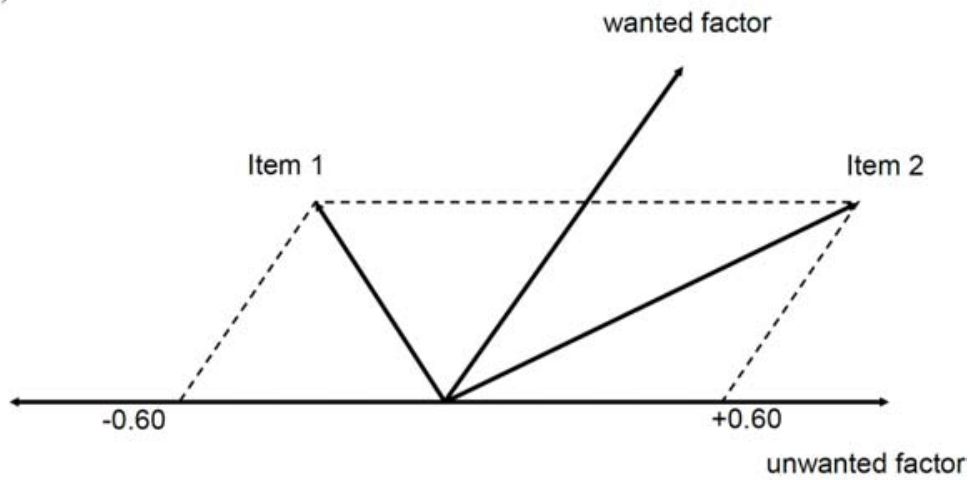

Figure 1. Example with two items loading positively on the wanted factor and loading with reversed sign and the same magnitude on the wanted and unwanted factor (A), with a different magnitude on the wanted and unwanted factor (B), and with same magnitude on the wanted and the oblique unwanted factor (C). 
Accordingly, an optimal suppression of unwanted variance could be described as

$$
\min _{l_{11} l_{12}, \ldots, l_{k 1} l_{k 2}}\left(\sum_{i=1}^{k} l_{i 1} l_{i 2}\right)^{2}
$$

where $l_{i 1}$ are the non-salient loadings on the first (wanted) factor, whereas $l_{i 2}$ describes the salient loadings on the second (unwanted) factor, which are used as the weights for the non-salient loadings of the first factor.

A balanced pattern of non-salient loadings can also occur in oblique factor solutions (Figure 1(C)). It can be seen from Figure 1(C) that a balanced pattern of positive and negative non-salient loadings can be achieved for oblique factor solutions even when a balanced pattern of non-salient loadings cannot be achieved in the corresponding orthogonal solution. A balanced pattern of positive and negative non-salient loadings will be termed a 'buffered simple structure' in the following.

The aim of the present study is to propose and explore the buffered simple structure as an alternative to the ICM. First, a more complete description of buffered simple structure is presented. Then, methods for defining buffered simple structure by means of CFA are proposed and illustrated by means of a simulated population example. In order to compare the ICM with buffered simple structure a small simulation study was performed.

\subsection{Buffered simple structure}

It should be noted that the specification of the non-salient loadings according to the minimum in Equation (2) implies for a $p$ variables $\times q$ factors loading matrix $\mathbf{L}$ that $\mathbf{L}^{\prime} \mathbf{L}$ is a diagonal matrix for a two-factor 
model $(q=2)$. It is therefore possible to define buffered simple structure (BS) as

$$
\mathbf{B S}=\min _{\mathbf{L}}\left(\mathbf{1}^{\prime}\left(\mathbf{L}^{\prime} \mathbf{L}-\operatorname{diag}\left(\mathbf{L}^{\prime} \mathbf{L}\right)\right)^{\circ 2} \mathbf{1}\right), \quad \text { for } q \leq 2,
$$

where 1 is a unit column vector of order $q \times 1$, “2” indicates the elementwise power, and $\mathbf{L}$ the pattern of factor loadings. Equation (3) yields zero if $\mathbf{L}^{\prime} \mathbf{L}=\operatorname{diag}\left(\mathbf{L}^{\prime} \mathbf{L}\right)$. A balanced set of positive and negative non-salient loadings implies that the weighted sum of the non-salient loadings corresponding to each subset of variables with salient loadings on one factor is a minimum. However, for $q>2$ buffered simple structure does not imply $\mathbf{L}^{\prime} \mathbf{L}=\operatorname{diag}\left(\mathbf{L}^{\prime} \mathbf{L}\right)$ for the columns of non-salient loadings. An example for a three-factor loading pattern $\mathbf{L}$ composed by three submatrices or blocks $\mathbf{B}_{i}$, each comprising a column $\mathbf{S}_{i}$ of three salient loadings and two columns $\mathbf{N}_{i j}$ of three non-salient loadings, is

$$
\mathbf{L}=\left[\begin{array}{l}
\mathbf{B}_{1} \\
\mathbf{B}_{2} \\
\mathbf{B}_{3}
\end{array}\right]=\left[\begin{array}{ccc}
\mathbf{S}_{1} & \mathbf{N}_{11} & \mathbf{N}_{12} \\
\mathbf{N}_{21} & \mathbf{S}_{2} & \mathbf{N}_{22} \\
\mathbf{N}_{31} & \mathbf{N}_{32} & \mathbf{S}_{3}
\end{array}\right]=\left[\begin{array}{ccc}
\boldsymbol{s}_{11} & \boldsymbol{n}_{12} & \boldsymbol{n}_{13} \\
\boldsymbol{s}_{21} & \boldsymbol{n}_{22} & \boldsymbol{n}_{23} \\
\boldsymbol{s}_{31} & \boldsymbol{n}_{32} & \boldsymbol{n}_{33} \\
\boldsymbol{n}_{41} & \boldsymbol{s}_{42} & \boldsymbol{n}_{43} \\
\boldsymbol{n}_{51} & \boldsymbol{s}_{52} & \boldsymbol{n}_{53} \\
\boldsymbol{n}_{61} & \boldsymbol{s}_{62} & \boldsymbol{n}_{63} \\
\boldsymbol{n}_{71} & \boldsymbol{n}_{72} & \boldsymbol{s}_{73} \\
\boldsymbol{n}_{81} & \boldsymbol{n}_{82} & \boldsymbol{s}_{83} \\
\boldsymbol{n}_{91} & \boldsymbol{n}_{92} & \boldsymbol{s}_{93}
\end{array}\right] .
$$

In order to achieve a buffered simple structure with respect to each factor, it is necessary to define for each of the three blocks of the matrix presented in Equation (4) that 


$$
\min _{\mathbf{B}_{i}}\left(\left(\mathbf{S}_{1}^{\prime} \mathbf{N}_{11}\right)^{\circ 2}+\left(\mathbf{S}_{1}^{\prime} \mathbf{N}_{12}\right)^{\circ 2}+\left(\mathbf{S}_{2}^{\prime} \mathbf{N}_{21}\right)^{\circ 2}+\left(\mathbf{S}_{2}^{\prime} \mathbf{N}_{22}\right)^{\circ 2}+\left(\mathbf{S}_{3}^{\prime} \mathbf{N}_{31}\right)^{\circ 2}+\left(\mathbf{S}_{3}^{\prime} \mathbf{N}_{32}\right)^{\circ 2}\right),
$$

where " 2 " denotes the element-wise power (and the subscripts are based on the matrix in the middle of Equation (4)). For the complete loading matrix $\mathbf{L}$ the minimum is

$$
\min _{\mathbf{L}}\left(\operatorname{tr}\left(\left(\mathbf{L}_{s}^{\prime} \mathbf{L}-\operatorname{diag}\left(\mathbf{L}_{s}^{\prime} \mathbf{L}\right)\right)^{\prime}\left(\mathbf{L}_{s}^{\prime} \mathbf{L}-\operatorname{diag}\left(\mathbf{L}_{s}^{\prime} \mathbf{L}\right)\right)\right)\right),
$$

with $\mathbf{L}_{s}$ as a matrix that contains only the salient loadings of $\mathbf{L}$ and where "tr" denotes the trace. If $\mathbf{L}_{s}^{\prime} \mathbf{L}=\operatorname{diag}\left(\mathbf{L}_{s}^{\prime} \mathbf{L}\right)$ is obtained, the right hand side of Equation (6) is zero. For the ICM Equation (6) is zero, because $\mathbf{L}_{s}^{\prime} \mathbf{L}=\operatorname{diag}\left(\mathbf{L}_{s}^{\prime} \mathbf{L}\right)$ when all non-salient loadings are zero (which implies $\left.\mathbf{L}=\mathbf{L}_{s}\right)$. It is, however, possible to obtain $\mathbf{L}_{s}{ }^{\prime} \mathbf{L}=\operatorname{diag}\left(\mathbf{L}_{s}{ }^{\prime} \mathbf{L}\right)$, even when the non-salient loadings are not zero, because buffered simple structure only requires that for each block the sum of non-salient loadings weighted by the salient loadings of the block is zero.

Equation (6) can also become zero if $\mathbf{L}_{s}=\mathbf{0}$. It is therefore necessary to provide a method that excludes that the absolute size of $\mathbf{L}_{s}$ is minimized. One method is to start from an ICM with substantial salient loadings and to insert these salient loadings as fixed values into Equation (6). Another method is to provide a condition that excludes a minimization of the absolute size of the salient loadings by means of defining a matrix

$$
\mathbf{H}_{s}= \begin{cases}\mathbf{L}_{s}^{\circ 2}+1, & \text { if } \mathbf{L}_{s}^{\circ 2}>0 \\ 0 & \text { else. }\end{cases}
$$


Then, the matrix $\mathbf{H}_{s}$ can be used for

$$
\min _{\mathbf{L}}\left(\operatorname{tr}\left(\left(\mathbf{H}_{s}^{\prime} \mathbf{L}-\operatorname{diag}\left(\mathbf{H}_{s}^{\prime} \mathbf{L}\right)\right)^{\prime}\left(\mathbf{H}_{s}^{\prime} \mathbf{L}-\operatorname{diag}\left(\mathbf{H}_{s}^{\prime} \mathbf{L}\right)\right)\right)\right)
$$

Since the salient loadings are the largest absolute loadings, it is extremely unlikely that the salient loadings become exactly zero. Accordingly, the weights for the salient loadings will be greater one, when $\mathbf{H}_{s}$ is used instead of $\mathbf{L}_{s}$ as a weight matrix. Obviously, a zero value for Equation (8) can only be obtained by means of a weighted sum of positive and negative non-salient loadings in $\mathbf{L}$ or if the non-salient loadings in $\mathbf{L}$ are zero. Using the squared salient loadings in Equation (7) excludes that a minimum is reached for a combination of positive and negative salient loadings. Accordingly, minimizing Equation (8) implies that a buffered simple structure is found that does not lead to small absolute salient loadings. When this condition is used, it is not necessary to start with an initial ICM so that a single modelling step is sufficient for obtaining a buffered simple structure. Therefore, this procedure is termed the 'one-step' procedure in the following. If researchers regard the buffered simple structure more as a refinement of an initial ICM, they will probably opt for the successive modelling procedure. If researchers consider a priori that the ICM is an overly restrictive model for their data, they can use a single modelling step based on Equation (8).

\subsection{Finding buffered simple structure}

The conditions expressed in the previous section can be used as a basis for CFA when they are specified as model constraints. An example for the one-step modelling based on model constraints corresponding to Equation (8) is provided in the next section. The modelling steps that are necessary when an ICM is used as a starting model for the multi-step procedure are summarized in Table 1 . In a first step, the salient loadings are to be estimated in the ICM (Model 1). In a second step, the salient loadings of Model 1 are used as weights for the weighted sum of the non- 
salient loadings for each block of salient loadings. These weighted sums are constrained to be zero in order to specify and estimate the corresponding buffered simple structure model while the salient loadings are again freely estimated (Model 2). If the salient loadings estimated in Model 2 diverge substantially from the salient loadings used as initial weights in the model constraint, the weights in the model constraints should be replaced by the salient loadings of Model 2, and the model should again be estimated (Model 3). This procedure can be repeated until the salient loadings estimated from the model correspond exactly to the salient loadings from the previous model used as weights for the model constraints. An advantage of the initial ICM estimation is that the factor inter-correlations can be fixed according to the results of the ICM. This and the use of the salient loadings in the model constraint assures that the definition of the salient loadings of the initial ICM is not substantially altered by the buffered simple structure of the non-salient loadings. An example for a corresponding Mplus syntax is presented together with the following population example. 
Table 1. Multi-step procedure for the estimation of buffered simple structure by means of CFA

\begin{tabular}{|c|c|c|c|c|}
\hline $\begin{array}{l}\text { Step } \\
\text { (Model) }\end{array}$ & Salient loadings & $\begin{array}{l}\text { Factor inter- } \\
\text { correlations }\end{array}$ & $\begin{array}{l}\text { Non-salient } \\
\text { loadings }\end{array}$ & Model constraint \\
\hline Model 1 & Free estimation & Free estimation & Fixed to zero & - \\
\hline Model 2 & Free estimation & $\begin{array}{l}\text { Fixed to ICM } \\
\text { values }\end{array}$ & Free estimation & $\begin{array}{l}\text { The sum of the } \\
\text { nonsalient loadings } \\
\text { weighted by the } \\
\text { salient loadings from } \\
\text { Model } 1 \text { is zero }\end{array}$ \\
\hline Model 3 & Free estimation & $\begin{array}{l}\text { Fixed to ICM } \\
\text { values }\end{array}$ & Free estimation & $\begin{array}{l}\text { The sum of the non- } \\
\text { salient loadings } \\
\text { weighted by the } \\
\text { salient loadings from } \\
\text { Model } 2 \text { is zero }\end{array}$ \\
\hline \multicolumn{5}{|c|}{$\begin{array}{l}\text { Repeat } i \text { times until the weights in the model constraint and the salient loadings resulting } \\
\text { from free estimation are equal: }\end{array}$} \\
\hline $\begin{array}{l}\text { Model } \\
(3+i)\end{array}$ & Free estimation & $\begin{array}{l}\text { Fixed to ICM } \\
\text { values }\end{array}$ & Free estimation & $\begin{array}{l}\text { The sum of the } \\
\text { nonsalient loadings } \\
\text { weighted by the } \\
\text { salient loadings from } \\
\text { Model }(2+i) \text { is zero }\end{array}$ \\
\hline
\end{tabular}

\subsection{Population example: Buffered simple structure}

The loading matrix in Table 2 has a perfect buffered simple structure, because the sum of the non-salient loadings multiplied by the salient loadings is zero for each block of salient loadings, i.e., the positive and negative non-salient loadings are perfectly balanced. The population correlation matrix generated from the population model presented in Table 2 was submitted to CFA (Mplus 7.11; Muthén \& Muthén, 19982013). The first estimation of buffered simple structure was based on the one-step modelling according to Equation (8). The complete model specification (Mplus syntax) containing the corresponding model constraint for each column of the loading matrix can be found in Appendix A. Since no sample size information is relevant for the population data, the fit is only evaluated by means of the standardized 
root mean squared residual (SRMR). The model fits almost perfectly to the data (SRMR $=.002)$ and the model estimates obtained for this model correspond exactly to the estimates obtained in Model 3 as well as to the population model (see Table 2). In order to illustrate the multi-step procedure, an ICM was estimated by means of maximum likelihood estimation. Salient loadings were freely estimated, non-salient loadings were fixed to zero, factor variances were fixed to one, and factor intercorrelations were freely estimated. The ICM model fits already quite well to the data $($ SRMR $=.073)$. The resulting salient loading estimates (Model 1, see Table 2) were used as weights for the model constraints imposed on the secondary loadings when Model 2 was estimated and the factor inter-correlations were fixed according to the initial ICM. The model specification and the model constraints are presented in Appendix A. Model 2 fits almost perfectly to the data $(\mathrm{SRMR}=.002)$ and the resulting parameter estimates presented in Table 2 are nearly identical to the parameters of the population model. However, the salient loadings used for the constraints (.594) were not identical to the salient loadings estimated for Model 2 (.600). Therefore, a final model based on the salient loading estimates of Model 2 as weights in the model constraints was calculated (Model 3). However, this final step does not alter the parameter estimates and model fit, so that no additional columns were presented in Table 2. The only difference between Model 2 and Model 3 is that the salient loadings used in the model constraints condition (.600) are identical to the salient loading estimates of Model 3. Thus, three steps were necessary in order to obtain a buffered simple structure by means of an initial ICM (Model 1: ICM, Model 2: model constraints, Model 3: adjusted model constraints).

In order to illustrate the difference between the buffered simple structure obtained by means of CFA with a more conventional procedure, a specification search starting from Model 1 (ICM) was performed (see Table 2). Modification indices for Model 1 were inspected and for each factor three non-salient loadings with the largest modification indices 
(> 15) were freely estimated (Model 4). A larger number of freed modification indices would lead to collapsing factors or to identification problems. The resulting Model 4 had an acceptable fit (SRMR $=.059)$. The loading pattern shows quite substantial non-salient loadings, especially on the second factor. Moreover, the loading size of the salient loadings is quite different in Model 4, although the salient loadings were of equal size in Model 1 as well as in Model 2 and 3. This demonstrates that substantial differences in the variables representing the factors are induced by the ICM with subsequent specification search, although these differences were not present in the population model. This shows that the ICM specification search strategy can be misleading when the data correspond to a buffered simple structure. 
Table 2. CFA-example: Population parameters, parameter estimates for ICM (Model 1), buffered simple structure (Model 2/3), and model resulting from specification search (Model 4)

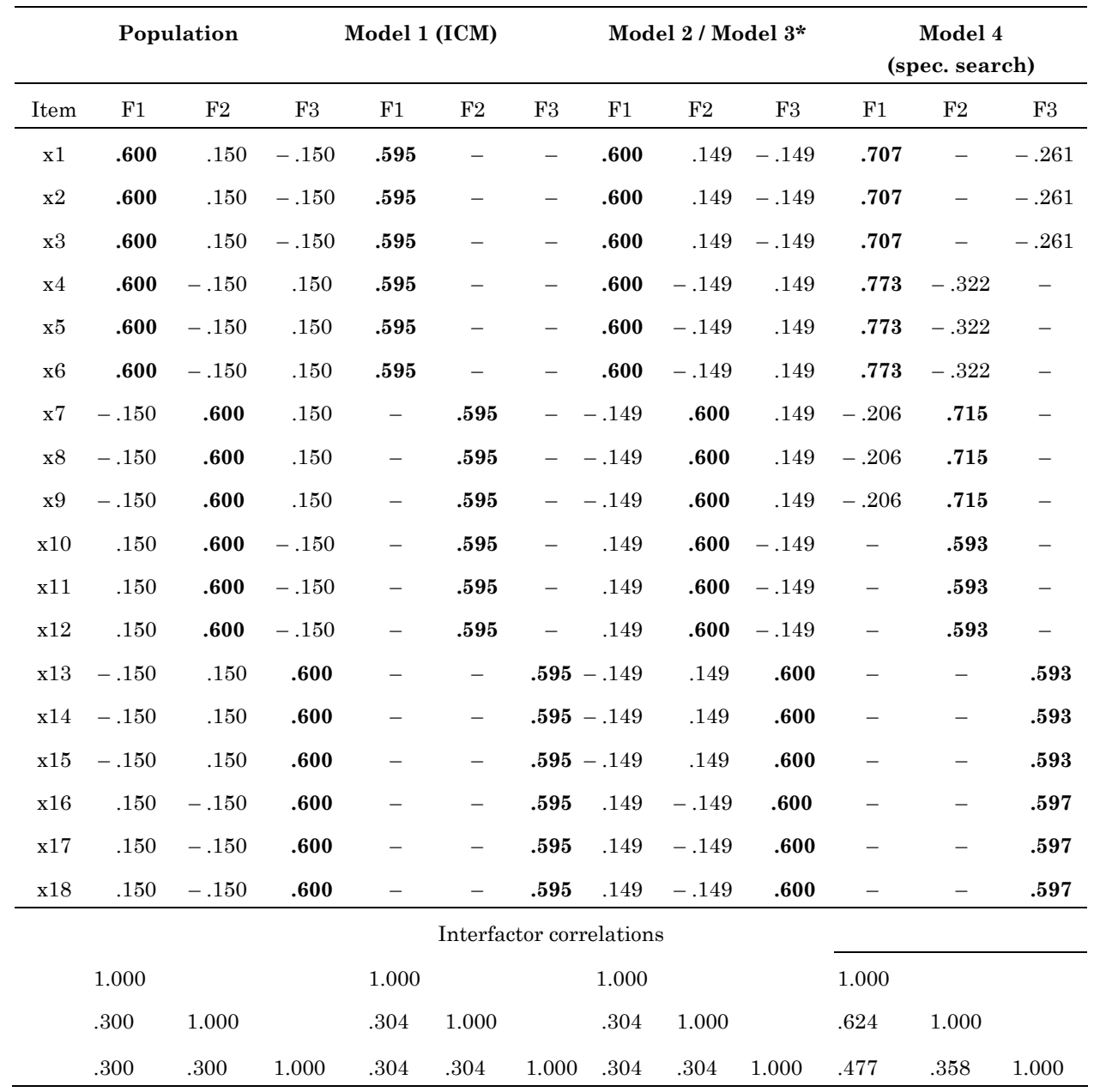

Note: Salient loadings are given in bold face. * The result obtained for Model 2 and Model 3 can also be obtained directly by means of the one-step procedure based on Equation (8) (see Appendix A). 


\section{Simulation Study}

Does the model constraint for the specification of buffered simple structure lead to a distortion of the salient loading estimates when the non-salient loadings are zero in the population? This question was investigated by means of a simulation study. Since buffered simple structure allows for the ICM as a special case, it is expected that the model constraints for buffered simple structure lead to precise salient loading estimates for population models corresponding to the ICM as well as for population models corresponding to buffered simple structure. Accordingly, the ICM was used as a population model for data generation as well as models with larger secondary loadings that correspond to a buffered simple structure.

Population models with six salient loadings per factor $(p / q)$ with three factors $(q=3)$, moderate $(l=.60)$ and large $(l=.80)$ salient loadings, with uncorrelated and slightly correlated factors (.30), and with a non-salient loading size of .00 were investigated as models with perfect simple structure (ICM). Models with buffered simple structure were generated as follows: For models with $l=.60$, models with an absolute non-salient loading size $(a n l)$ of .05 , as well as models with $a n l=.10, .15$, and .20 were generated. The same set of models was generated for $l=.80$. The signs of the non-salient loadings were distributed in a way that results in a perfect buffered simple structure. Thus, the condition $\mathbf{L}_{s}{ }^{\prime} \mathbf{L}=\operatorname{diag}\left(\mathbf{L}_{s}{ }^{\prime} \mathbf{L}\right)$ that was introduced in Equation (6) was zero for these population models. The buffered simple structure models were generated for orthogonal factors as well as for slightly correlated factors.

All models based on anl $=.00$ are ICM and all models based on $a n l=.05, .10, .15$, and .20 are buffered simple structures. Each of the (2 salient loading sizes $\times 5$ non-salient loading sizes $\times 2$ degrees of interfactor-correlations $\Rightarrow 20$ population models was investigated in 
1,000 samples with $n=150,300$, and 900 cases. The root mean squared difference (RMSD) between the population loadings and the corresponding estimated loadings was averaged across the 1,000 samples per condition. For the orthogonal population models, the interfactorcorrelations were fixed to zero for model estimation, whereas for the population models with interfactor-correlations of .30, the interfactorcorrelations were set free for model estimation in the samples.

For all conditions the mean root mean square error of approximation (RMSEA) of the estimated buffered simple structure models was below .05 , indicating an acceptable model fit. For the estimated ICM the mean RMSEA was greater .05 for all conditions with anl $\geq .10$, indicating that the ICM has a moderate or poor fit under these conditions. The RMSD for the loadings of the three-factor models are presented in Figure 2 (A)-(D) and the RMSD for the correlations of the models with correlated factors are presented in Figure 3 (A)-(B). The results for the conditions representing the ICM (with zero absolute non-salient population loadings; $a n l=.00)$ and for buffered simple structure $(a n l>.00)$ are presented together. When the population model is an ICM $(a n l=.00)$, the RMSD of the salient loadings was nearly same when an ICM or a buffered simple structure was specified (see Figure 2). 
(A) $l=.60$, interfactor-correlation $=.00$

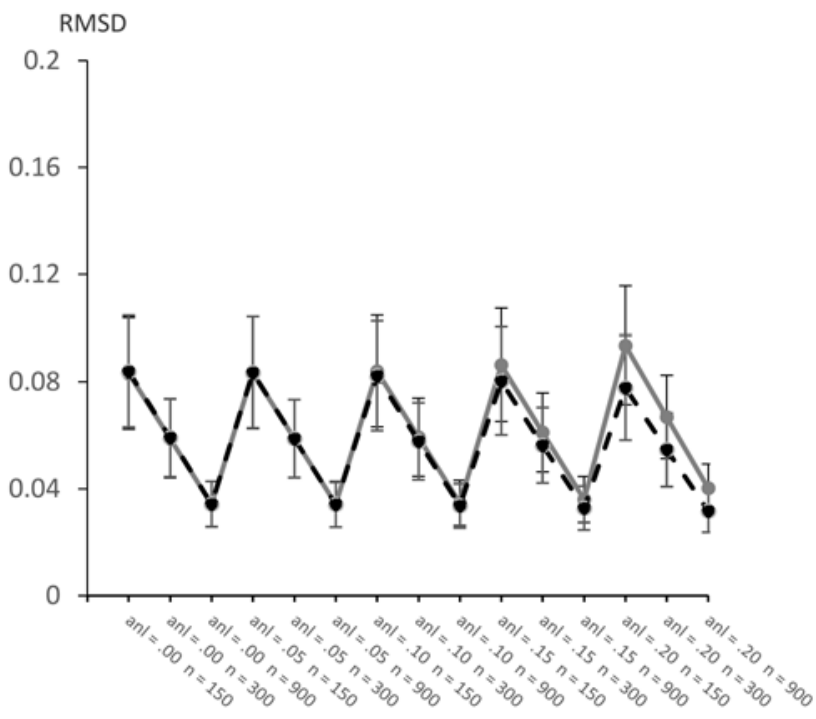

(B) $l=.60$, interfactor-correlation $=.30$

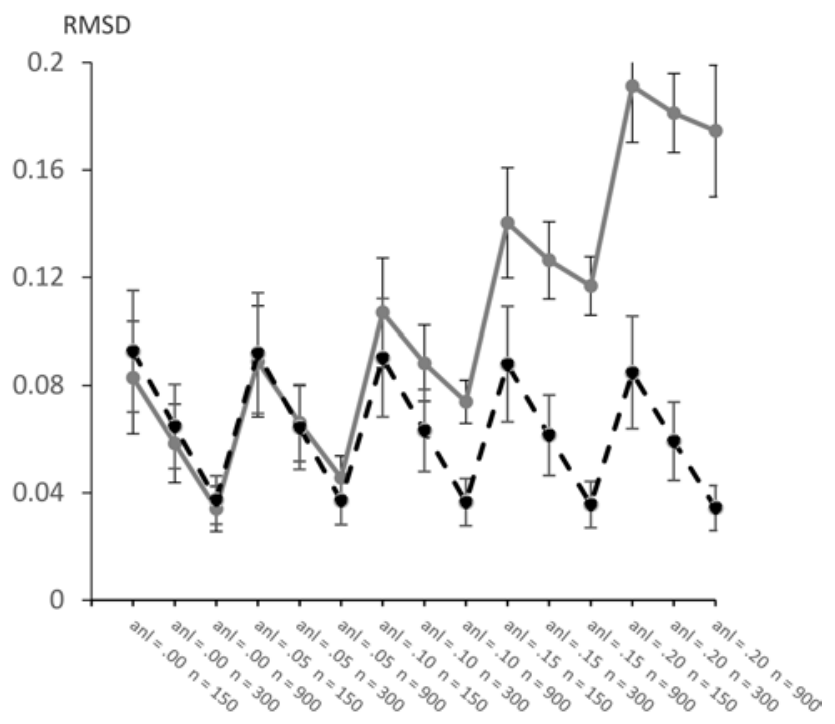


(C) $l=.80$, interfactor-correlation $=.00$

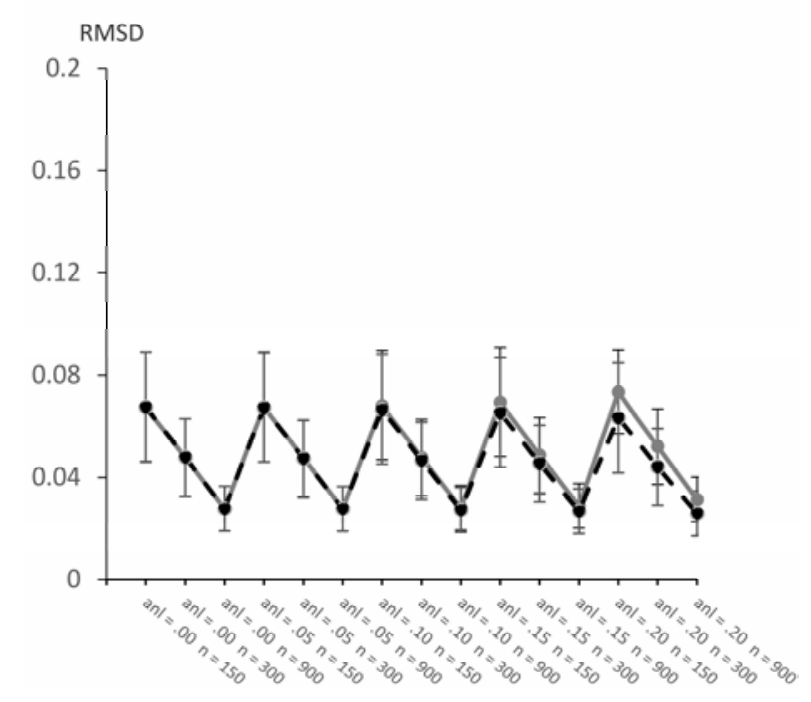

(D) $l=.80$, interfactor-correlation $=.30$

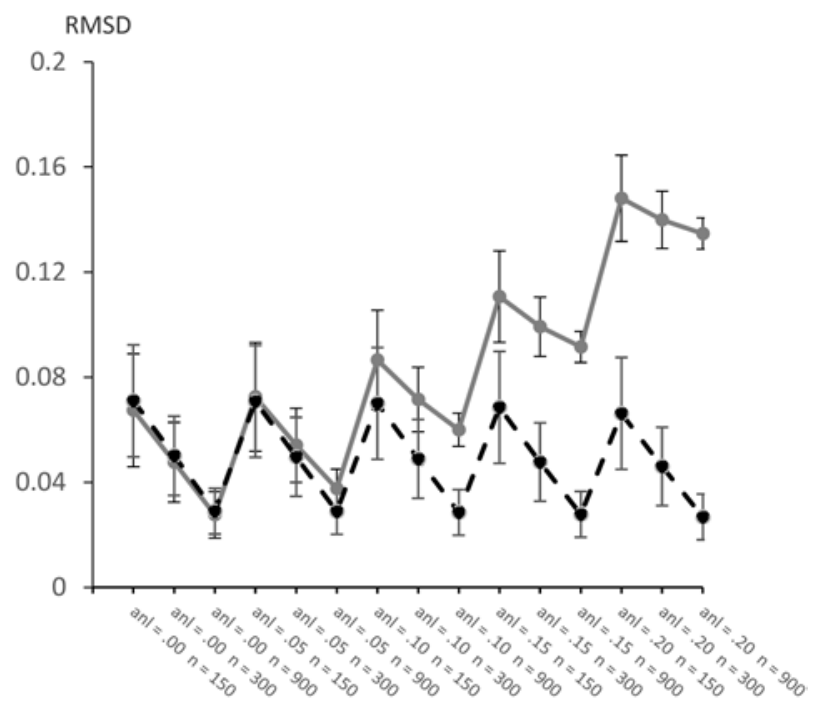

Figure 2. Root mean squared difference (RMSD) between population loadings and estimated sample loadings (error bars represent standard errors); $l=$ salient loadings; anl = absolute non-salient loadings; and $n=$ sample size. 
However, with increasing anl in the population, the RMSD of the salient loadings based on the specification of the ICM increased, whereas the RMSD based on buffered simple structure remained nearly constant. Since the RMSD for $l=.60$ and $n=150$ and correlated factors was slightly larger for the buffered simple structure than for the ICM, it is recommended to estimate an initial ICM when the sample size is small and when non-zero interfactor-correlations as well as small salient loadings are to be expected.

As for the factor loadings, the precision of reproducing the population interfactor-correlations was assessed by means of the mean squared difference (RMSD) between the population interfactor-correlations and the corresponding interfactor-correlations estimated from the samples (see Figure 3). 
(A) $l=.60$, interfactor-correlation $=.30$

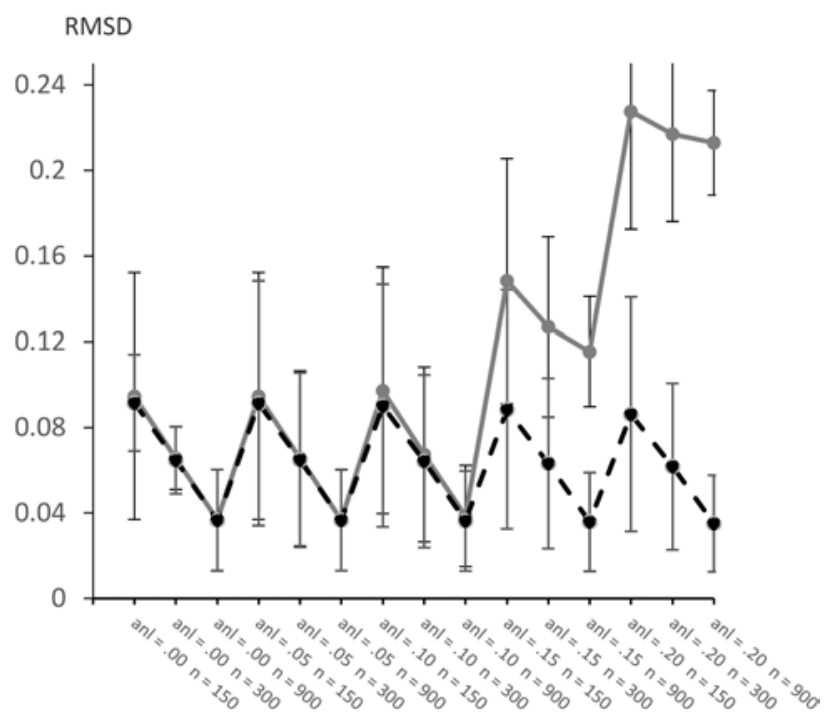

(B) $l=.80$, interfactor-correlation $=.30$

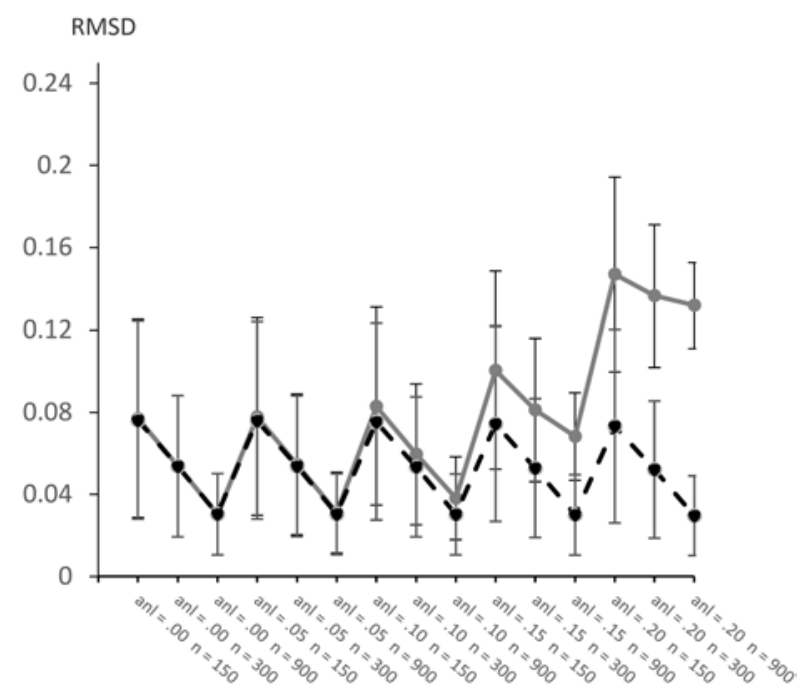

Figure 3. Root mean squared difference (RMSD) between population interfactor-correlations and estimated sample interfactor-correlations (error bars represent standard errors); $l=$ salient loadings; $a n l=$ absolute non-salient loadings; and $n=$ sample size. 
As for the loadings, no relevant differences between model estimation based on the ICM and model estimation based on buffered simple structure occurred for anl $=.00$, whereas the RMSD of ICM estimation increased with increasing anl. The RMSD for model estimation based on buffered simple structure remained nearly constant.

\section{Discussion}

An alternative criterion for the specification of factor loadings in CFA was proposed. The motivation for proposing this criterion was that the ICM is often overly restrictive. The main idea is to minimize the weighted sum of secondary or non-salient loadings. A minimal weighted sum of non-salient loadings can be obtained when the absolute size of the non-salient loadings is close to zero, which corresponds to simple structure or to the ICM. However, a minimal weighted sum can also be obtained when the absolute size of non-salient loadings is substantial and when the positive and negative non-salient weighted loadings are balanced out. Since this criterion contains simple structure as a special case and since it is based on the idea of buffered unwanted variance, it was named 'buffered simple structure'.

It was shown that buffered simple structure implies that for each block of variables with salient loadings, the corresponding sum of nonsalient loadings weighted by the salient loadings should be zero. It was shown that it is possible to specify buffered simple structure by means of

model constraints in CFA models. Two methods for the specification of buffered simple structure by means of CFA have been proposed. The first method uses a model constraint that avoids that buffered simple structure is reached through minimizing the absolute size of the salient loadings. This procedure is based on a single model (one-step procedure). The second method is based on the salient loading estimates of an initial ICM that are entered into a model constraint for subsequent models. Thus, when an initial ICM does not fit to the data, it is possible to 
estimate a buffered simple structure model with model constraints based on the salient loadings of the ICM. This illustrates that buffered simple structure can be conceived as an alternative to the ICM with subsequent specification searches.

A population loading matrix with buffered simple structure was used in order to show that a perfect buffered simple structure can be found by means of the one-step procedure as well as by means of an initial ICM followed by two additional CFA-models (multi-step procedure). It was shown for the multi-step procedure that a wrong assignment of variables on the model-constraint will lead to model rejection when the factor intercorrelations are fixed to the values of the initial ICM. It was also illustrated in the example that the strategy of estimating an ICM and to perform subsequent specification search for the loadings can be misleading when the data correspond to a buffered simple structure, because differences between loadings are induced where loadings were equal in the population.

A simulation study was performed in order to compare the precision of estimated population loadings and interfactor-correlations for the ICM and buffered simple structure models. It was found that buffered simple structure model estimates had a similar error as the ICM estimates when the population model was an ICM and when the sample size was at least moderate $(n=300)$. However, for the combination of small samples with small salient loadings samples and non-zero interfactor-correlations, the salient loadings of a population ICM were more precisely identified by means of an ICM. It is therefore recommended to estimate an initial ICM under these conditions and to proceed with subsequent model constraints (see Table 1) under these conditions. In contrast, when the population model had a buffered simple structure, the ICM parameter estimates were less precise than the parameter estimates based on buffered simple structure models. 


\section{References}

[1] R. B. Cattell and B. Tsujioka, The importance of factor-trueness and validity, versus homogeneity and orthogonality, in test scales, Educational and Psychological Measurement 24(1) (1964), 3-30.

doi: $10.1177 / 001316446402400101$

[2] W. K. B. Hofstee, B. De Raad and L. R. Goldberg, Integration of the big five and the circumplex approaches to trait structure, Journal of Personality and Social Psychology 63 (1992), 146-163.

doi: 10.1037/0022-3514.63.1.146

[3] O. Vassend and A. Skrondal, Validation of the NEO personality inventory and the five-factor model: Can findings from exploratory and confirmatory factor analysis be reconciled? European Journal of Personality 11 (1997), 147-166.

doi: 10.1002/(SICI)1099-0984(199706) 


\section{Appendix A}

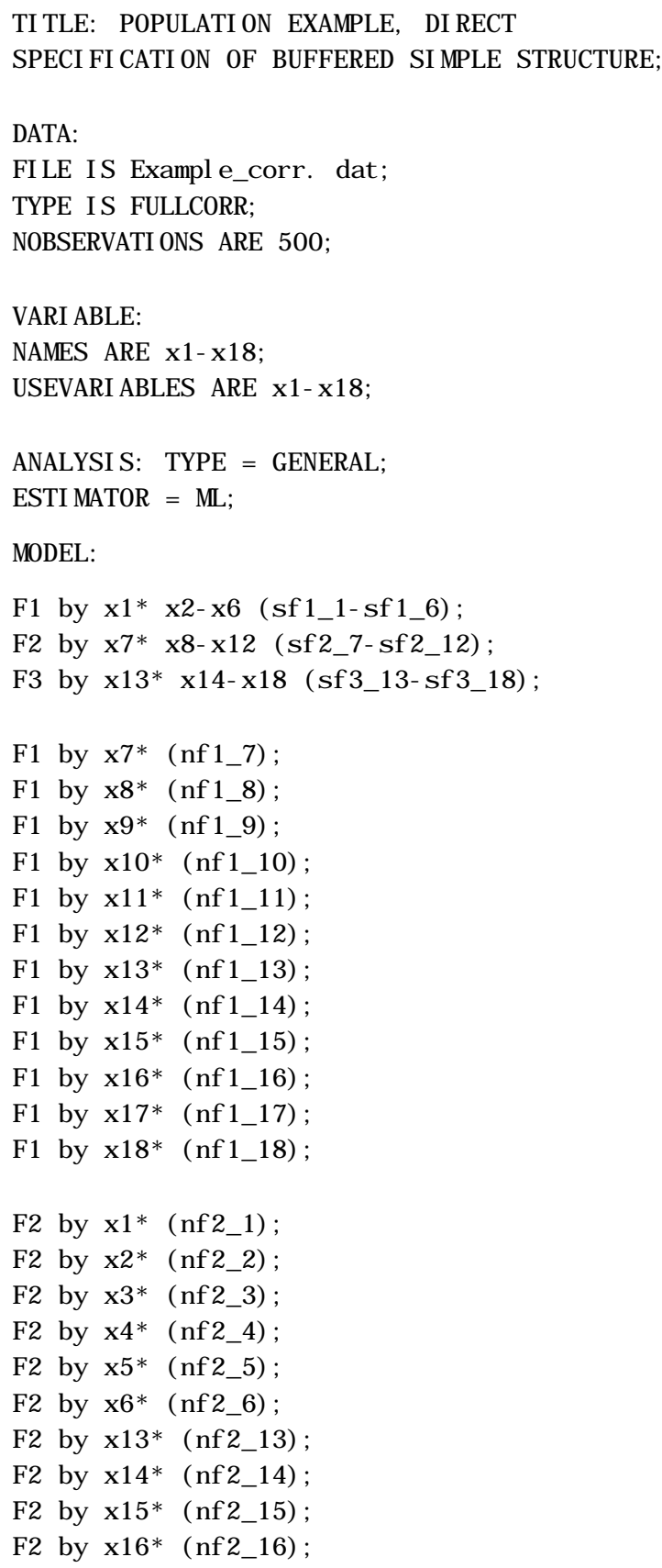


$\mathrm{F} 2$ by $\times 17^{*}\left(\mathrm{nf} 2 \mathrm{n}_{-} 17\right)$;

F 2 by $x 18 *(n f 2,18)$;

F 3 by $x 1^{*}\left(n f 3_{-} 1\right)$;

$\mathrm{F} 3$ by $\times 2 *\left(\mathrm{nf} 3_{-} 2\right)$;

$\mathrm{F} 3$ by $\times 3^{*}\left(\mathrm{nf} 33_{-} 3\right)$;

$F 3$ by $x 4^{*}(n f 3-4)$;

$\mathrm{F} 3$ by $x 5^{*}\left(\mathrm{nf} 3{ }_{-} 5\right)$;

$F 3$ by $x 6^{*}\left(\mathrm{nf} 3 \_6\right)$;

$F 3$ by $x 7^{*}\left(\operatorname{nf} 3_{-} 7\right)$;

$F 3$ by $x 8 *\left(n f 3 \_8\right)$;

F 3 by $\times 9 *(n f 3-9)$;

$\mathrm{F} 3$ by $x 10 *(n f \overline{3}-10)$;

$F 3$ by $x 11^{*}\left(n f 3 \_11\right)$;

F 3 by $\times 12^{*}\left(n f 3 \_12\right)$;

F $1 @ 1 ; \quad F 2 @ 1 ; \quad F 3 @ 1 ;$

MODEL CONSTRAINT:

$$
\begin{aligned}
& 0=\left(\left(1+s f 1_{-} 1 * * 2\right) * n f 2_{-} 1+\left(1+s f 1_{-} 1 * * 2\right) * n f 2_{-} 2+\left(1+s f 1_{-} 1 * * 2\right) * n f 2_{-} 3\right. \\
& \left.+\left(1+s f 1_{-} 4 * * 2\right) * n f 2_{-} 4+\left(1+s f 1_{-} 5 * 2\right) * n f 2_{-} 5+\left(1+s f 1_{-} 6 * 2\right) * n f 2_{-} 6\right) * * 2 \text {; } \\
& 0=\left(\left(1+s f 1_{-} 1 * * 2\right) * n f 3_{-} 1+\left(1+s f 1_{-} 1 * * 2\right) * n f 3_{-} 2+\left(1+s f 1_{-} 1 * * 2\right) * n f 3_{-} 3\right. \\
& \left.+\left(1+s f 1_{-} 4 * 2\right) * n f 3_{-} 4+\left(1+s f 1_{-} 5 * * 2\right) * n f 3_{-} 5+\left(1+s f 1_{-} 6 * 2\right) * n f 3_{-} 6\right) * * 2 ; \\
& 0=\left(\left(1+s f 2_{-} 7 * 2\right) * n f 1_{-} 7+\left(1+5 f 2_{-} 8 * * 2\right) * n f 1_{-} 8+\left(1+5 f 2_{-} g * 2\right) * n f 1_{-} g\right. \\
& \left.+\left(1+s f 2_{-} 10 * * 2\right) * n f 1_{-} 10+\left(1+s f 2_{-} 11 * * 2\right) * n f 1_{-} 11+\left(1+s f 2_{-} 12 * * 2\right) * n f 1_{-} 12\right) * * 2 \text {; } \\
& 0=\left(\left(1+s f 2_{-} 7 * 2\right) * n f 3_{-} 7+\left(1+s f 2_{-} 8 * * 2\right) * n f 3_{-} 8+\left(1+s f 2_{-} g * 2\right) * n f 3_{-} g\right. \\
& \left.+\left(1+5 f 2_{-} 10 * * 2\right) * n f 3_{-} 10+\left(1+5 f 2_{-} 11 * * 2\right) * n f 3_{-} 11+\left(1+5 f 2_{-} 12 * * 2\right) * n f 3_{-} 12\right) * * 2 \text {; } \\
& 0=\left(\left(1+s f 3_{-} 13 * * 2\right) * n f 1_{-} 13+\left(1+5 f 3_{-} 14 * 2\right) * n f 1_{-} 14+\left(1+5 f 3_{-} 15 * * 2\right) * n f 1_{-} 15\right. \\
& \left.+\left(1+5 f 3 \_16 * 2\right) * n f 1_{-} 16+\left(1+5 f 3_{-} 17 * * 2\right) * n f 1_{-} 17+\left(1+5 f 3_{-} 18 * * 2\right) * n f 1_{-} 18\right) * * 2 ; \\
& 0=\left(\left(1+s f 3_{-} 13 * * 2\right) * n f 2_{-} 13+\left(1+s f 3_{-} 14 * * 2\right) * n f 2_{-} 14+\left(1+s f 3_{-} 15 * * 2\right) * n f 2_{-} 15\right. \\
& \left.+\left(1+5 f 3_{-} 16 * 2\right) * n f 2_{-} 16+\left(1+5 f 3_{-} 17 * 2\right) * n f 2_{-} 17+\left(1+5 f 3_{-} 18 * * 2\right) * n f 2_{-} 18\right) * * 2 \text {; }
\end{aligned}
$$

OUTPUT: 


\section{Appendix B}

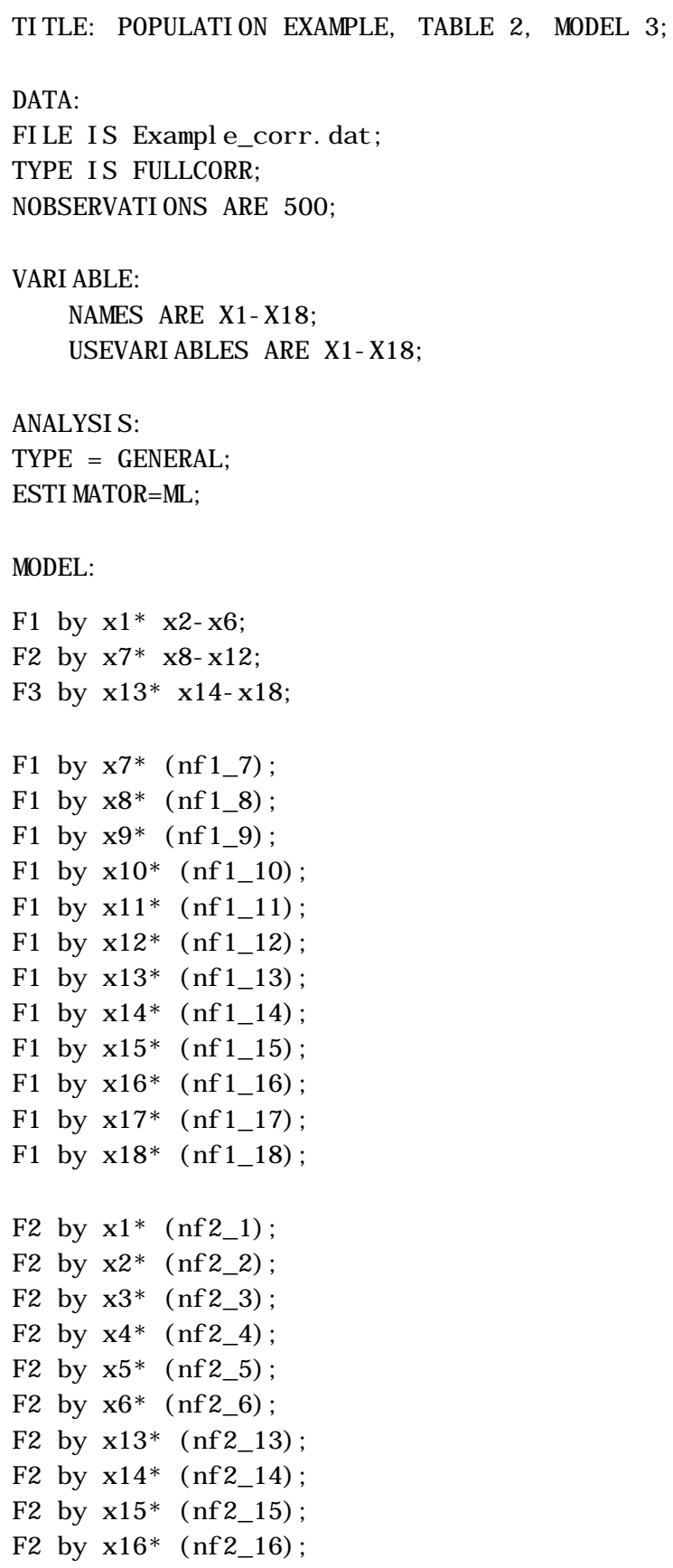




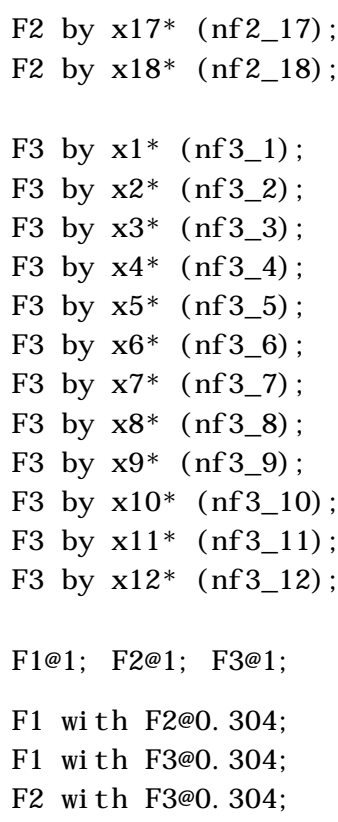

